\title{
Topologically protected states in a spider web lattice
}

\author{
J. P. Pino $\odot,{ }^{1,2}$ P. Alves, ${ }^{1}$ J. D. Gouveia $\odot,{ }^{3}$ A. M. Marques, ${ }^{1}$ and R. G. Dias ${ }^{1, *}$ \\ ${ }^{1}$ Departamento de Física \& I3N, Universidade de Aveiro, Campus Universitário de Santiago, 3810-193 Aveiro, Portugal \\ ${ }^{2}$ Departamento de Física \& CIDMA, Universidade de Aveiro, Campus Universitário de Santiago, 3810-193 Aveiro, Portugal \\ ${ }^{3}$ Departamento de Física \& CICECO, Universidade de Aveiro, Campus Universitário de Santiago, 3810-193 Aveiro, Portugal
}

(Received 29 June 2020; accepted 3 November 2020; published 8 December 2020)

\begin{abstract}
Topological phases and edge-state topological protection are explicitly related by the bulk-edge correspondence which assumes the existence a real space boundary in one-dimensional (1D), 2D, or 3D systems. Extensions of this picture to systems where the boundary is present in the domain of generalized coordinates may generate unusual topological behavior in real space. In this paper, we discuss topological radial states in molecules with rotational symmetry and staggered hopping amplitudes in the radial direction. In the absence of the central site, the model is characterized as a weak topological insulator supporting radial edge states in the topological phase. When the central site is considered, we show that it behaves as an effective perturbation coupled in different ways to the radial inner edge, depending on the type of orbital considered at this central site. Tuning the parameters of the central orbital allows for a precise control over the radial inner edge physics of the model. We further break rotational symmetry by studying sliced molecules, which creates open boundaries in the azimuthal direction also, and show that higher-order boundary (corner) modes are present and how they can be driven into an energy gap through coupling with the central orbital. The results are corroborated by numerical conductance studies.
\end{abstract}

DOI: 10.1103/PhysRevResearch.2.043337

\section{INTRODUCTION}

The bulk-boundary correspondence in topological Hamiltonians of independent particles tells us that nontrivial bulk topological phases have a one-to-one relation with the presence of topologically protected edge states in these systems when a real space boundary is considered [1]. This correspondence can be obviously generalized to the case of more complex Hamiltonians that can be mapped onto these topological Hamiltonians. In this mapping, the degrees of freedom that correspond to the real space coordinates of the topological Hamiltonian can be very diverse and the mapped boundary in the original degrees of freedom may translate into unusual real space behavior. One example of such a mapping is that of a two-particle interacting ring where the relative position of two spinless fermions becomes a degree of freedom with open boundaries (due to the Pauli exclusion principle) where a topological edge state can be observed (without any real space boundary) [2].

Presently, several methods of constructing artificial lattices are experimentally accessible that allow one to test the behavior of topological models. These include for example ultracold atoms in optical lattices [3], photonic crystals [4],

\footnotetext{
"To whom correspondence should be addressed: rdias@ua.pt
}

Published by the American Physical Society under the terms of the Creative Commons Attribution 4.0 International license. Further distribution of this work must maintain attribution to the author $(s)$ and the published article's title, journal citation, and DOI. topolectrical circuits [5-8], acoustic lattices [9,10], vacancy lattices in a chlorine monolayer on a copper surface [11], or a lattice generated by an array of atoms or molecules positioned on a surface with a scanning tunneling microscope [12]. These methods allow one to construct lattices with arbitrary geometry as well as the control over the tight-binding parameters associated with the artificial lattice.

For example, in the case of ultracold quantum gases in optical lattices, it is possible to control tunneling terms as well as onsite energies (modifying the potential wells) and even interactions strengths (using Feshbach resonances, see Ref. [13]).

Most two-dimensional (2D) lattice geometries should be easily realizable using these artificial systems, but of course, some lattice geometries may increase the difficulty in generating the desired tunneling parameters. Some of these platforms offer advantages in that regard. For example, topolectrical circuits would offer a privileged venue for the simulation of the weblike lattices studied in our paper [6,8]. Indeed, arbitrary lattice geometries can be realized in these systems, with the hopping and on-site potential parameters dependent only on the capacitance and inductance values of the capacitors and inductors used, respectively, and not on the physical distance between nodes (sites), that is, no decay in the hopping values is expected along the azimuthal direction as the radius increases.

Several authors have suggested more complex realizations of topological models in these artificial lattices such as models with an extra degree of freedom at each lattice site (for example, diamond chain lattice filled with ultracold atoms loaded into finite angular momentum states [14]) and interacting topological models [15]. 


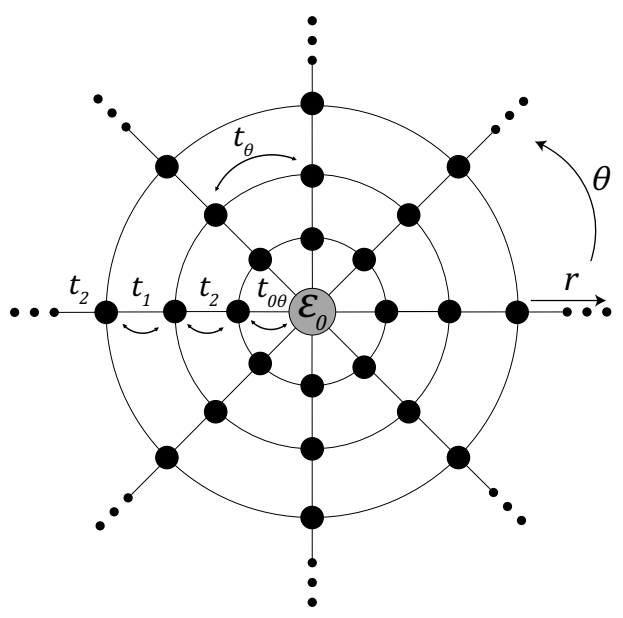

(a)



(b)

FIG. 1. (a) Tight binding with a central site with a on-site potential $\epsilon$ and alternating hopping parameters $t_{1}$ and $t_{2}$ along the $r$ coordinate. Along the azimuthal direction, we have a hopping parameter $t$. (b) Schematic representation of the same model, assuming now the situation where the central site is a $p_{x}$ orbital.

The model that we address in this paper has simple boundaries when using polar coordinates and should be easily realizable using the methods mentioned above. More precisely, we study a one-particle tight-binding model of a finite 2D cluster with discrete rotational symmetry with staggered hopping terms along the radial directions (see Fig. 1). The possibility of staggered hopping terms along the azimuthal directions is also addressed. The one-particle eigenstates of this model can describe qualitatively, for example, the onemagnon subspace of high-spin organic molecules [16] that have been extensively researched having in mind the development of novel magnetic materials $[17,18]$. We show that this model has topologically protected states which are localized at the center of the molecule reflecting the respective lower boundary of the radial coordinate and the model can be classified as a weak topological insulator. However, the central site and in particular the symmetry of the relevant orbital, introduces subtleties in this topological characterization which cannot be described using a single weak topological invariant.

The paper is organized in the following way. In Sec. II, we present the tight-binding Hamiltonian of our weblike lattice, where different angular momentum states ( $s, p$, and $d$ orbitals) for the central site are considered and analyzed. The bulk energy dispersion relations are computed for a simple case. In Sec. III, boundary effects are discussed and a mapping from the weblike model onto a system of decoupled 1D chains is performed. In Sec. III A, numerical simulations are carried out, where we compute the energy spectrum as a function of the on-site potential of the central site for all three cases of angular momentum states and where we show the observable edge states in the system. In Sec. III B, the exact eigenstates for our weblike system are determined for the scenarios of $s$ and $p$ orbitals. Comparisons between analytical solutions and its corresponding numerical counterpart are detailed. Then, in Sec. IV, we discuss the conductance profiles as a function of chemical potential for various scenarios of angular momentum states, on-site potentials for the central site and contacts, as a way to showcase conductance features due to the central "edge" states in the model. In Sec. V, we discuss the possibility of existence of corner states, in the case of a sliced version of the original 2D model. Finally, in Sec. VI, we provide the conclusions.

\section{MODEL}

We start our study by introducing our simple weblike model with discrete rotational symmetry: a set of concentric rings with hopping terms between them, see Fig. 1(a). We assume that the number of sites in each ring is the same so that the respective tight-binding Hamiltonian is

$$
\begin{aligned}
\hat{H}= & \sum_{r=1}^{N_{r}-1} \sum_{\theta=1}^{N_{\theta}}\left(-t_{r} \hat{c}_{r+1, \theta}^{\dagger} \hat{c}_{r, \theta}-t_{\theta} \hat{c}_{r, \theta+1}^{\dagger} \hat{c}_{r, \theta}+\text { H.c. }\right) \\
& +\sum_{\theta=1}^{N_{\theta}}\left(-t_{0 \theta} \hat{c}_{1, \theta}^{\dagger} \hat{c}_{0}+\text { H.c. }\right)+\varepsilon_{0} \hat{c}_{0}^{\dagger} \hat{c}_{0},
\end{aligned}
$$

where $N_{r}$ and $N_{\theta}$ represent respectively the number of sites along the radial direction (denoted by the integer index label $r$ ) and the number of sites in each ring (along the azimuthal direction denoted by the index $\theta$ ); $t_{r}=t_{1}\left(t_{2}\right)$ for $r$ even (odd) represents hoppings along the radial direction; $t_{\theta}$ the hopping along the azimuthal direction; $t_{0 \theta}$ is a hopping between the central site and the adjacent ones, with numerical dependencies with the angle $\theta$, in accordance with the central site angular momentum orbital, as we show later; and $\epsilon_{0}$ is an onsite potential for the central site. $\hat{c}_{r, \theta}$ and $\hat{c}_{r, \theta}^{\dagger}$ are destruction and creation operators, respectively, whose index represents its site in $(r, \theta)$ coordinates. Periodic boundary conditions are considered in the azimuthal direction $\left(N_{\theta}+1 \equiv 1\right)$. Our system can be seen as a combination of a quantum ring in the azimuthal direction and a quantum chain with open boundary conditions in the radial direction, that is, a cylinder (see Fig. 2) if we discard the central site. We consider staggered hopping terms in the radial direction and uniform azimuthal hopping terms $t_{\theta}=t$, as shown in Fig. 1(a). In Sec. V, the hopping parameters $t_{\theta}$ are also staggered.

The hopping terms to the central site, $t_{0 \theta}$, depend on the type of orbital we assume for the central atom ( $s$ orbitals are assumed for all other sites, as a simplification). In order to address the differences in the case of $s, p$, and $d$ orbitals at the central site (a gate potential applied at the central site allows one to go from one to another in an experiment), our $t_{0 \theta}$ parameters follow the expressions by Slater and Koster [19]. Let us look at each case separately. For a $s$ orbital central atom, the parameters $t_{0 \theta}$ are constant, due to the spherical symmetry 




FIG. 2. Schematic representation of the transformation from the our 2D weblike structure to a 3D cylinder in the case $N_{\theta}=4$. The red cube indicates the sites within the unit cell of our structure.

of the $s$ orbital. For $p$ orbitals, more specifically, $p_{x}$, we now have to consider angular dependence of the hopping parameters, that is, $t_{0 \theta_{j}}=\cos \left(\theta_{j}\right) V_{s p \sigma}$, with $\theta_{j}=\frac{2 \pi}{N_{\theta}} j$ and $V_{s p \sigma}$ a constant. In the case of our weblike lattice, one can then write $t_{0 \theta} / V_{s p \sigma}=\left(e^{i \frac{2 \pi j}{N_{\theta}}}+e^{-i \frac{2 \pi j}{N_{\theta}}}\right) / 2$. A similar argument can then be followed for $d$ orbitals. For the case of $d_{x^{2}+y^{2}}$, the angular dependence is expressed as $t_{0 \theta_{j}}=\frac{\sqrt{3}}{2}\left[\cos ^{2}(\theta)-\sin ^{2}(\theta)\right] V_{s d \sigma}$, which leads to $t_{0 \theta} / V_{s d \sigma}=\frac{\sqrt{3}}{4}\left(e^{i \frac{4 \pi j}{N_{\theta}}}+e^{-i \frac{4 \pi j}{N_{\theta}}}\right)$. In order to simplify the notation, in previous and following equations, we identify $\theta$ with $j$ in $\theta_{j}=\frac{2 \pi}{N_{\theta}} j$, see Fig. 1(b).

The model is block diagonal in the azimuthal Bloch basis,

$$
\begin{aligned}
\hat{H}_{k_{\theta}}^{\text {orb }}= & \varepsilon_{0} \hat{c}_{0}^{\dagger} \hat{c}_{0}+2 \cos \left(k_{\theta}\right) \sum_{r=1}^{N_{r}} \hat{c}_{r, k_{\theta}}^{\dagger} \hat{c}_{r, k_{\theta}} \\
& +\sum_{r=1}^{N_{r}}\left(-t_{r} \hat{c}_{r+1, k_{\theta}}^{\dagger} \hat{c}_{r, k_{\theta}}+\text { H.c. }\right) \\
& +t_{0 \theta} N_{\theta}^{1 / 2}\left[\delta_{\text {orb }, s} \hat{c}_{1, k_{\theta}=0}^{\dagger} \hat{c}_{0}\right. \\
& +\delta_{\text {orb }, p}\left(\hat{c}_{1, k_{\theta}=\frac{2 \pi}{N_{\theta}}}^{\dagger} \hat{c}_{0}+\hat{c}_{1, k_{\theta}=-\frac{2 \pi}{N_{\theta}}}^{\dagger} \hat{c}_{0}\right) \\
& +\delta_{\text {orb }, d} \frac{\sqrt{3}}{4}\left(\hat{c}_{1, k_{\theta}=\frac{4 \pi}{N_{\theta}}}^{\dagger} \hat{c}_{0}+\hat{c}_{1, k_{\theta}=-\frac{4 \pi}{N_{\theta}}}^{\dagger} \hat{c}_{0}\right) \\
& + \text { H.c. }],
\end{aligned}
$$

where $t_{\theta}=1$ was set as the energy unit and $k_{\theta}$ is the azimuthal momentum (which can be interpreted as the angular momentum), with quantization condition $k_{\theta}=\frac{2 \pi}{N_{\theta}} n, n=0, \ldots, N_{\theta}-$ 1. The Kronecker deltas are defined as

$$
\delta_{\text {orb }, j}= \begin{cases}0, & \text { if orb } \neq j, \\ 1, & \text { if orb }=j,\end{cases}
$$

for each combination of angular momentum orbitals, i.e., orb $=j=s, p, d$. One can readily observe that the Hamiltonian $\hat{H}_{k_{\theta}}$ describes a SSH model, except in the case of $k_{\theta}=0, \pm \frac{2 \pi}{N_{\theta}}$ and $\pm \frac{4 \pi}{N_{\theta}}$ when the central site has respectively a $s, p$, and $d$ orbital.

We will now consider the simple case $N_{\theta}=4$ (the generalization to arbitrary $N_{\theta}$ is trivial). In this case, we may redraw our weblike 2D lattice as 3D lattice with an unit cell of eight sites, as seen in Fig. 2 (red cube). We can write the respective bulk Hamiltonian $H_{k_{r}}$ in the basis

$$
\begin{aligned}
& \left\{A_{k_{r}}, B_{k_{r}}, C_{k_{r}}, D_{k_{r}}, A_{k_{r}}^{*}, B_{k_{r}}^{*}, C_{k_{r}}^{*}, D_{k_{r}}^{*}\right\} \text { as } \\
& \hat{H}\left(k_{r}\right)=\left[\begin{array}{cc}
\alpha & \beta \\
\beta^{\dagger} & \alpha
\end{array}\right], \quad \text { with } \quad \alpha=-\left[\begin{array}{cccc}
0 & t_{1} & 0 & t_{1} \\
t_{1} & 0 & t_{1} & 0 \\
0 & t_{1} & 0 & t_{1} \\
t_{1} & 0 & t_{1} & 0
\end{array}\right]
\end{aligned}
$$

and

$\beta=-\left[\begin{array}{cccc}t_{1}+t_{2} e^{-i k_{r}} & 0 & 0 & 0 \\ 0 & t_{1}+t_{2} e^{-i k_{r}} & 0 & 0 \\ 0 & 0 & t_{1}+t_{2} e^{-i k_{r}} & 0 \\ 0 & 0 & 0 & t_{1}+t_{2} e^{-i k_{r}}\end{array}\right]$,

with dispersion relations, $E_{1 \pm}= \pm \Delta, E_{2 \pm}=2 t_{1} \pm$ $\Delta, E_{2 \pm}=-2 t_{1} \pm \Delta$, where $\Delta=\sqrt{t_{1}^{2}+t_{2}^{2}+2 t_{1} t_{2} \cos \left(k_{r}\right)}$.

\section{EDGE MODES AND TOPOLOGICAI CHARACTERIZATION}

In this section, we address the boundary effects present in a finite weblike lattice. It is important to note that in the radial direction one has two boundaries, an external and an internal one. The internal one corresponds to the center of the molecule and as we show below, the topological characterization of our system will depend on the type of orbital of the central atom in the molecule. Note that when we state which orbital is present at the central site, it is implicit that the other orbitals of the central site are energetically distant of the energies of the $s$ orbitals of the rest of the lattice and therefore we may ignore them in the Hamiltonian and in the topological characterization of our model.

Let us consider a finite web structure of concentric four-site rings (as shown in the left diagram of Fig. 2). On Fourier transforming over the periodic azimuthal direction, the system is a combination of four different Hamiltonians, $\hat{H}=$ $\hat{H}_{k_{\theta}=0}+\hat{H}_{k_{\theta}=\pi}+\hat{H}_{k_{\theta}=\frac{\pi}{2}}+\hat{H}_{k_{\theta}=-\frac{\pi}{2}}$. Each Hamiltonian represents a chain with open boundary conditions, as shown in Figs. 3(a)-3(c). Our system should behave similarly to a set of four Su-Schrieffer-Heeger (SSH) chains [20]. However, the existence of the central site leads to an interesting topological behavior. In order to characterize this behavior, one must recall that the SSH chain (with integer number of unit cells) has topological edge states (reflecting a bulk topological phase with a nonzero winding number) when the chain has a weak link at both ends. This simple topological characterization of the SSH chain can be extended to higher-dimensional models, one of the simplest examples being a ribbon of dimers (coupled chains of dimers) [21]. Such a system supports dispersive edge states along the dimer direction (when open boundaries are present in that direction) and their respective energies can be found from the Hamiltonian obtained on Fourier transforming in the direction perpendicular to the dimerization [21]. This system of weakly coupled staggered chains constitutes an example of a weak topological insulator with edge states in the dimer direction only [22-24]. The Hamiltonian given by Eq. (2), except for terms that involve the central site, describes such a ribbon of dimers in the coordinates $\left(r, k_{\theta}\right)$ in the limit of zero interchain coupling, as illustrated in Figs. 3(a), 3(b), and $3(\mathrm{c})$. 


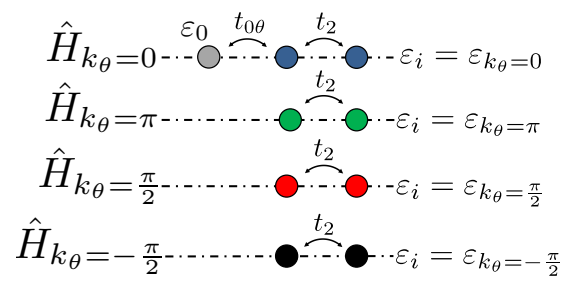

(a)

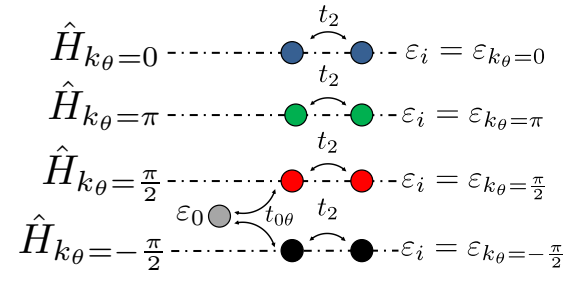

(b)



(c)

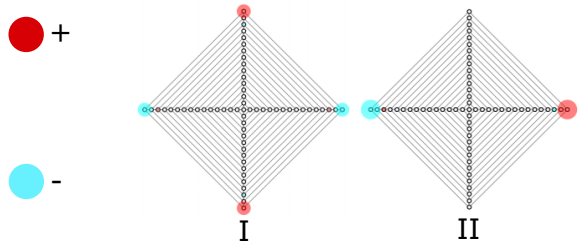

(d)

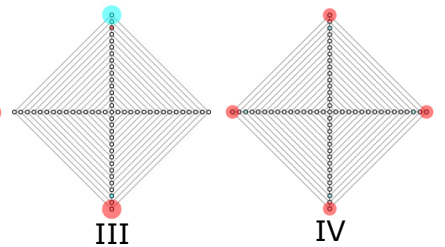

III

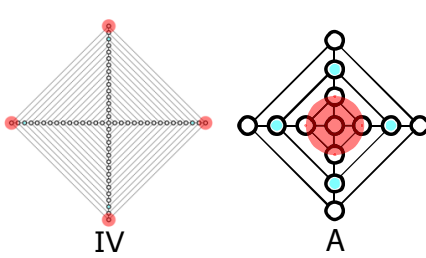

A

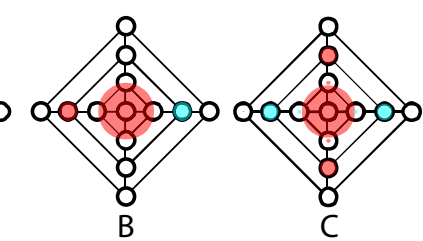

(e)

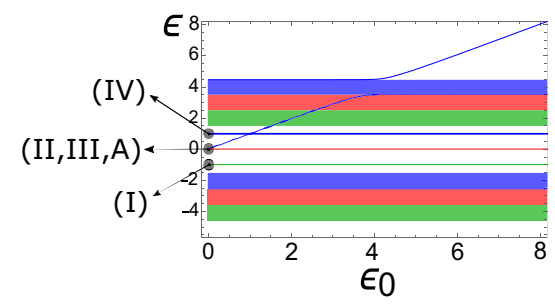

(f)

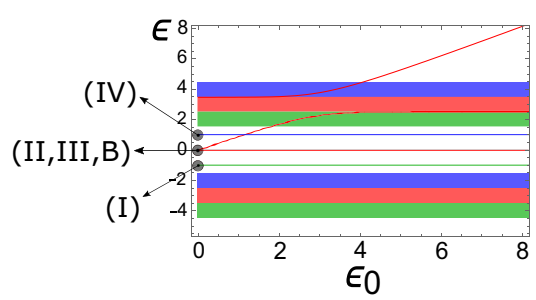

(g)

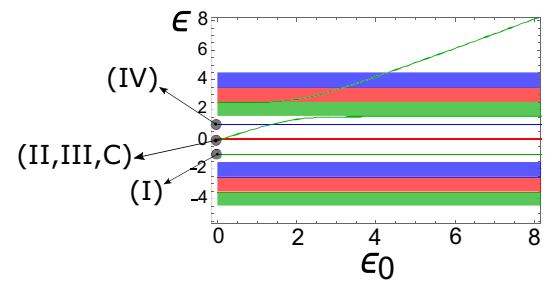

(h)

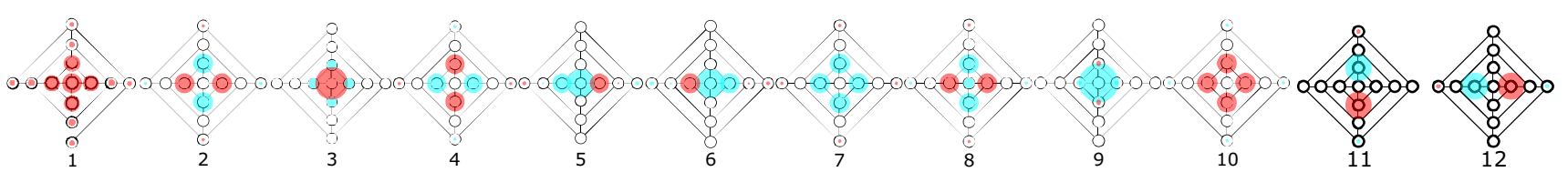

(i)

(j)

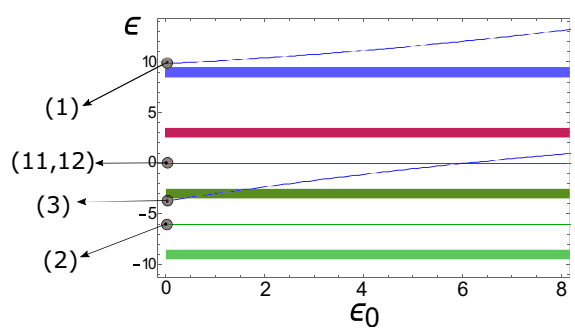

$(\mathrm{k})$



(1)

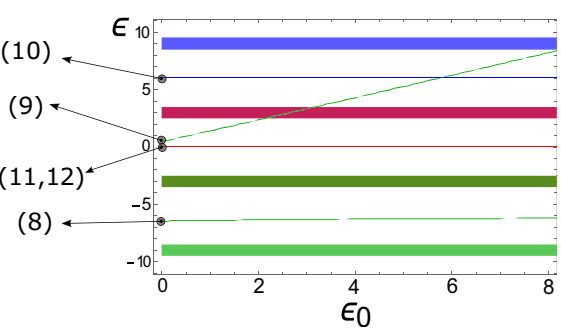

(m)

FIG. 3. Effective chains corresponding to $\hat{H}_{k_{\theta}=0}, \hat{H}_{k_{\theta}=\pi}, \hat{H}_{k_{\theta}=\frac{\pi}{2}}$, and $\hat{H}_{k_{\theta}=-\frac{\pi}{2}}$ for the case of a web structure with four sites per ring with the central site being (a) $s$ orbital, (b) $p$ orbital, and (c) $d$ orbital, for $V_{s d \sigma}=1$. (d) Schematic representation of the four edge states present in the case of $\varepsilon_{0}=0$ and $t_{2}=3$ and $t_{1}=0.5$, all four states are present for the three different scenarios where we have an $s$ orbital, $p$ orbital, and $d$ orbital at the central site. (e) Schematic representation of the central edge states present in the case of $\varepsilon_{0}=0$ and $t_{2}=3$ and $t_{1}=0.5$, with (A) $s$ orbital, (B) $p$ orbital, and (C) $d$ orbital. [(f)-(h)] Energy dispersion as functions of $\varepsilon_{0}$ in the case of a (f) $s$ orbital, (g) $p$ orbital, and (h) $d$ orbital at the central site and assuming 15 rings in the web structure for $t_{2}=3$ and $t_{1}=0.5$. [(i) and (j)] Schematic representation of the edge states present in the case of $\varepsilon_{0}=0$ and $t_{2}=0.5$ and $t_{1}=3$, where we have an $s$ orbital $(1,2,3,11,12), p$ orbital $(4,5,6,7,11,12)$, and $d$ orbital $(9,10,11,12)$ at the central site. $[(\mathrm{k})-(\mathrm{m})]$ Energy dispersion as functions of $\varepsilon_{0}$ in the case of a (k) $s$ orbital, (l) $p$ orbital, and (m) $d$ orbital at the central site and assuming 15 rings in the web structure for $t_{2}=0.5$ and $t_{1}=3$.

The presence of the central site changes the topological behavior of such a ribbon since (i) adding one more site to the edge of an OBC SSH chain makes the topological edge state disappear or appear (the edge hopping term changes from a weak link to a strong one or vice versa), and (ii) if the extra site has an on-site potential different from those of the other sites of the chain, then as this potential is varied, one may observe a change of the topological edge state into an impurity edge state $[25,26]$. This implies that in the cases displayed in Fig. 3, we may observe "mixed" topological behavior (for example, three chains may be topologically trivial and one is not or vice versa) that changes depending on the central site orbital (the change between orbitals may be induced applying a gate potential at the central site). Note that therefore one may 
state that our weblike molecule falls into the weak topological insulators class but with these contrasting features that imply that one cannot characterize its behavior using a single weak topological invariant as usually assumed [22]. We discuss such behavior in the following subsections.

\section{A. Numerical results}

In Figs. 3(a), 3(b), and 3(c), we show the effective set of SSH chains with staggered hoppings $t_{1}$ and $t_{2}$ corresponding to $\hat{H}_{k_{\theta}=0}, \hat{H}_{k_{\theta}=\pi}, \hat{H}_{k_{\theta}=\frac{\pi}{2}}$, and $\hat{H}_{k_{\theta}=-\frac{\pi}{2}}$ for the case of a web structure with four sites per ring with the central site being respectively (a) an $s$ orbital, (b) a $p$ orbital, and (c) a $d$ orbital. The symmetry of the central site orbital determines to which effective chain the extra site is coupled. In particular, for a $p$ orbital, the extra site joins the $\hat{H}_{k_{\theta}=\frac{\pi}{2}}$ and $\hat{H}_{k_{\theta}=-\frac{\pi}{2}}$ chains. For a web structure with $N_{\theta}$ sites in each ring, one can conclude from the Hamiltonian given by Eq. (2) that the central site with an $s$ orbital, $p$ orbital, or $d$ orbital respectively introduces an extra site in the $\hat{H}_{k_{\theta}=0}$ chain, in the $\hat{H}_{k_{\theta}=4 \pi / N_{\theta}}$ chain and joins the $\hat{H}_{k_{\theta}=2 \pi / N_{\theta}}$ and $\hat{H}_{k_{\theta}=-2 \pi / N_{\theta}}$ chains. The hopping parameter from the extra site to the SSH chains depends on the symmetry of the central orbital [see Eq. (2)].

The behavior of the web structure reflects not only the central site local potential and orbital symmetry but also our choice of staggered hopping parameters as well as the number of rings in the web structure. In Figs. 3(d) and 3(e), one shows the outer and central edge states of the web structure assuming 15 rings in the web structure (besides the central site). Note that we designate the central localized states as edge states because that is what they are in the mapping shown in Figs. 3(a), 3(b), and 3(c). In the case of the 15 rings web structure, the effective SSH chains decoupled from the extra site have a noninteger number of unit cells and that implies that each of them has a single edge state, left (inner or central) or right (outer) depending on the ratio between the hopping parameters. For $t_{2}=3$ and $t_{1}=0.5$, these are outer edge states and one observes a single central edge or impurity state for any symmetry of the central orbital. However, the spectrum as a function of the central site potential reflects the orbital symmetry clearly. The color scheme of the bands and edge or impurity states in Figs. 3(f), 3(g), 3(h), 3(k), 3(1), and 3(m), indicates which chain in Figs. 3(a), 3(b) and 3(c) generates the bands and edge or impurity states. Since the red and black chains generate degenerate levels, only the red levels are shown. As one observes in Figs. 3(f), 3(g), and 3(h), the topological edge-state level merges with the respective bulk band at different energies as expected on the basis of the mapping onto the effective SSH chains. The same can be said for the impurity level.

\section{B. Analytical results}

The exact eigensolution of the weblike lattice can be reached using the mapping into the decoupled chains. In the following, we determine the eigenstates of the chains with the additional site. The chain with the impurity site has different energy levels, so the full solution cannot be written as a linear combination of these eigenstates with those of other decoupled chains, which are the usual eigenstates of the SSH chain.
When an impurity site is connected by $t_{0 \theta}$ at one edge to an SSH chain and has an on-site energy offset $\epsilon_{0}$, relative to the rest of the chain, the magnitude of the radial momentum values $k$ of the eigenstates in each of the $\lambda(k)=$ $\pm \sqrt{t_{1}^{2}+t_{2}^{2}+2 t_{1} t_{2} \cos (k)}$ energy bands is shifted by a $k$ dependent phase [25],

$$
\begin{gathered}
\phi_{\lambda}^{I}(k)=(N+1) k-n \pi, \quad n=1,2, \ldots, N, \\
\phi_{\lambda}^{I}(k)=\cot ^{-1}\left[\frac{1}{\alpha_{I}(\lambda) \sin k}+\cot k\right],
\end{gathered}
$$

where $N=\frac{N_{r}}{2}$ is the number of unit cells of the SSH chain and

$$
\alpha_{I}(\lambda)=\alpha_{\mathrm{SSH}}(\lambda)-\frac{\lambda t_{0 \theta}^{2}}{t_{1} t_{2}\left(\lambda-\epsilon_{0}\right)},
$$

with $\alpha_{\mathrm{SSH}}(\lambda)=\frac{t_{1}}{t_{2}}$ as the constant unperturbed parameter for both bands, that is, in the absence of the extra impurity site.

States with energy outside the bulk energy bands may also appear and this implies that the respective radial momenta become complex, $k=q+i p$ [21,26-29], where the real part is given by $q=0 \vee \pi$. The imaginary part $p$ of the complex radial momentum is the inverse localization length of the edge state and it is given by the solution to

$$
\begin{gathered}
\phi_{\lambda_{ \pm}}^{I}=(N+1) p, \\
\phi_{\lambda_{ \pm}}^{I}=\operatorname{coth}^{-1}\left[\frac{1}{ \pm \alpha_{I}\left(\lambda_{ \pm}\right) \sinh (p)}+\operatorname{coth}(p)\right],
\end{gathered}
$$

obtained from (5) and (6), respectively, by substituting $k \rightarrow$ $q+i p$ everywhere, which includes the energy bands $\lambda(k) \rightarrow$ $\lambda_{ \pm}(p)$, where $q=0(\pi)$ relates to the "+" ("-") solution (each bulk band originates two edge bands, distinguished by the real part of the radial momentum) [25]. Note that one only searches for solutions to (8) if the bulk solutions found with (5) are less than the total number of states, given in this case by $2 N+1$. We will now discuss how we can apply this formalism to our model, more precisely, to the Hamiltonian of the decoupled chains seen in Figs. 3(a), 3(b), and 3(c). We present here the results for the $s$-orbital and the $p$-orbital cases, since the $d$-orbital scenario results in a similar analysis to the $s$-orbital case.

For the mapped chains of the $s$-orbital case, shown in Fig. 3(a), the momentum values of the eigenstates are found by directly solving (5) and (8), using just $\alpha_{\mathrm{SSH}}(\lambda)$ in (7) for the unperturbed $k_{\theta}= \pm \frac{\pi}{2}, \pi$ chains, whereas the full $\alpha_{I}(\lambda)$ parameter is used for the $k_{\theta}=0$ chain, the only one connected to the impurity site. For the mapped chains of the $p$-orbital case, shown in Fig. 3(b), the solutions for the unperturbed $k_{\theta}=0, \pi$ chain are found by applying the same procedure as for the unperturbed chains of the $s$-orbital case. However, the impurity site is now connected to both the $k_{\theta}= \pm \frac{\pi}{2}$ chains. If we unfold and align these two chains, then we can see that we effectively have two SSH chains connected by a midchain impurity. We define a new basis spanned by the the bonding and antibonding pairs $|r, \pm\rangle=\frac{1}{\sqrt{2}}\left(\left|\frac{\pi}{2}, r\right\rangle \pm\left|-\frac{\pi}{2}, r\right\rangle\right)$, where $\left|k_{\theta}, r\right\rangle$ is the basis state of the $k_{\theta}$ chain at the radial position $r$, with $r=1,2, \ldots, N_{r}$. Note that the on-site potentials across both chains are the same, that is, $\epsilon_{k_{\theta}=\frac{\pi}{2}}=\epsilon_{k_{\theta}=-\frac{\pi}{2}}=0$. This 


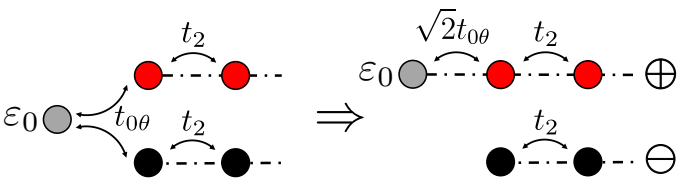

FIG. 4. Extra decoupling in the case of the $p$ orbital into chains of bonding and antibonding states, generating a SSH chain and a SSH chain connected to an extra site by a renormalized hopping $\sqrt{2} t_{0 \theta}$.

generates the two decoupled bonding and antibonding chains shown in Fig. 4. Crucially, the impurity site only couples to site $|1,+\rangle$ of the bonding chain with a renormalized hopping parameter. At this point, one finds the absolute radial momentum solutions for the unperturbed antibonding and perturbed bonding chains using same method as for the corresponding $s$-orbital cases, with the $t_{0 \theta} \rightarrow \sqrt{2} t_{0 \theta}$ substitution in the perturbed bonding chain. Finally, it is clear that the $d$-orbital case of Fig. 3(c) is equivalent to the $s$-orbital case of Fig. 3(a). Note, however, that the on-site potential at the impurity site is taken to be the energy offset in relation to the rest of the chain, such that the perturbed chain of the $s$-orbital case one has $\epsilon_{0} \rightarrow \epsilon_{0}-\epsilon_{k_{\theta}=0}$, while for the perturbed $d$-orbital case one has $\epsilon_{0} \rightarrow \epsilon_{0}-\epsilon_{k_{\theta}=\pi}$, with $\epsilon_{k_{\theta}=\pi}=-\epsilon_{k_{\theta}=0}=2 t$.

While the exact form of both the bulk and edge eigenstates for the unpertubed chains, which are just open SSH chains, is well known [21], the eigenstates for the perturbed chain can also be determined analytically [25]. Along the SSH chain sites, the (unnormalized) wave function is given by

$$
\left|\psi^{\sigma}(k)\right\rangle=\sum_{j=1}^{N}\left[\begin{array}{c}
\sin \left[k j-\phi_{\lambda}^{I}(k)\right] \\
\sigma \sin \left[k j-\phi_{\lambda}^{I}(k)+\phi_{\lambda}^{0}(k)\right]
\end{array}\right],
$$

where $\sigma= \pm$ indicates the corresponding energy band, $\lambda \rightarrow \lambda^{\sigma}(k)=\sigma \sqrt{t_{1}^{2}+t_{2}^{2}+2 t_{1} t_{2} \cos (k)}, \quad$ and $\quad \phi_{\lambda}^{0}(k)=$ $\phi_{\lambda}^{I}\left(t_{0, \theta}=0, \varepsilon_{0}=0\right)$. To find the amplitude at the impurity site, we apply the tight-binding eigenvalue equation to the impurity site,

$$
\psi_{0}^{\sigma}=-\frac{t_{0 \theta}}{\lambda^{\sigma}-\epsilon_{0}} \psi_{1, A}^{\sigma},
$$

where $\psi_{1, A}^{\sigma}=\sin \left[k-\phi_{\lambda}^{I}(k)\right]$ is the amplitude at the first site of the first unit cell. Finally, one gathers all the components (SSH chain + impurity site) and normalizes the state. The same procedure is followed in order to find the edge states, keeping in mind that their respective momenta are now complex $k=q+i p$, with $q=0 \vee \pi$. Numerical results for both bulk and edge momentum solutions, found by solving (5) and (8), respectively, for an SSH chain with $N=8$ unit cells connected to an impurity site and parameter choice $t_{1} / t_{2}=0.5$, $t_{0 \theta}=1$, and $\epsilon_{0}=1$ can be seen in Fig. 5. One less bulk solution in found for the negative band in Fig. 5(b), in relation to the positive band in Fig. 5(a). Since the total number of states is $2 N+1$ and $2 N-1$ bulk states are found, this indicates the existence of two edge states. The $p$ value of one of them, belonging to the edge band obtained from the $k \rightarrow i p$ substitution in the negative bulk band, is shown in Fig. 5(c) (the other edge solution, coming from the $k \rightarrow q+i p$ substitution in the positive bulk band, in not shown there). The wave function of



FIG. 5. Bulk phase shift $\phi_{\lambda}^{I}$ as a function of $k$ for the (a) positive and (b) negative $\lambda$ energy band, for an SSH chain with $N=8$ unit cells plus an extra impurity site. Equally spaced lines represent the successive $f_{n}(k)=(N+1) k-n \pi$, with $n=1,2, \ldots, N+1$. The $k$ values of the red dots at the intersections are the momentum values of the bulk eigenstates. (c) Plot of the purely real part of $\operatorname{coth}\left(\phi_{\lambda_{ \pm}}^{I}\right)$, where $\phi_{\lambda_{ \pm}}^{I}$ is the edge phase shift in (8) of the negative energy band, versus the imaginary part $p$ of the complex momentum $k=q \pm i p$, with $q=0(\pi)$ for the black (dark blue) curve. The $p$ value of the red dot at the intersection between the the curve for $q=0$ and the $\tanh (p(N+1))$ (light blue) curve is the imaginary component of the momentum of the edge eigenstate. (d) Spatial profile along the $j$ sites of the SSH chain plus an impurity at $j=0$ for one of the bulk $k$ states of the negative band in (b) and for the edge $p$ state found in (c), computed analytically through Eqs. (10) and (11) and verified numerically. Parameters: $t_{1} / t_{2}=0.5, t_{0 \theta}=1$ and $\epsilon_{0}=1$, in units of $t_{2}$.

the edge states and of one of the bulk states of the negative band is shown in Fig. 5(d).

After finding all the states for the mapped $k_{\theta}$ chains through the method outlined in this section, the states of the original weblike lattice can be determined simply by inverse Fourier transforming along the azimuthal direction. Ultimately, one arrives at three unperturbed $k_{\theta}$ chains for the different orbitals considered at the central site in Figs. 3(a)-3(c) (after rotating the basis for the $p$-orbital case, as depicted in Fig. 4). However, since the $k_{\theta}= \pm \frac{\pi}{2}$ chains have the same potential energy $\epsilon_{k_{\theta}= \pm \frac{\pi}{2}}=0$, only the $s$ - and $d$-orbital cases will exhibit an essential twofold degeneracy in the energy spectrum of the original lattice, for the subspace of states related to the unperturbed $k_{\theta}= \pm \frac{\pi}{2}$ chains.

\section{CONDUCTANCE PROFILES}

In the previous section, we have shown that the choice of the central orbital leads to specific central "edge" states. In this section, we show that the existence of the different central "edge" states leads to observable features in the conductance profiles for our simplified radial model. Our numerical calculations follow the approach in Ref. [30] for the conductance in the case of a tight-binding cluster connected to two semiinfinite tight-binding chains. 
The hoppings $t_{L}$ and $t_{R}$ are the left and right hopping parameters between the cluster and the contacts leads and generate finite transmission probability across the cluster. Since no two-particle interactions are considered in this paper, the transmission probability $\left|t\left(\epsilon_{k}\right)\right|^{2}$ for an incident particle with momentum $k$ and energy $\epsilon_{k}=-2 \cos (k)$ can be calculated using quantum scattering theory and is given by the following expression [30]:

$$
\begin{aligned}
\left|t\left(\epsilon_{k}\right)\right|^{2}= & 4 t_{L}^{2} t_{R}^{2} \sin ^{2} k \mid\langle R|\left[\epsilon_{k} \hat{I}_{s}-H_{s}\right. \\
& \left.+e^{i k}\left(t_{L}^{2}|L\rangle\left\langle L\left|+t_{R}^{2}\right| R\right\rangle\langle R|\right)\right]\left.^{-1}|L\rangle\right|^{2},
\end{aligned}
$$

where the inverse is to be found within the subspace of the cluster sites positions. $\hat{I}_{s}$ is the identity operator in that subspace and $\hat{H}_{s}$ a generic single-particle Hamiltonian at the scattering region. That is, finding the transmission probability has been reduced to finding a correlation in the cluster and apart from two onsite corrections at the sites of the cluster in contact with the leads, the leads play no further role in the calculation. This result can be partially explained in the following way. Quantum scattering theory states that, since the state $\left|\psi_{k}+\right\rangle$ of a particle with momentum $k$ and energy $\epsilon_{k}$ very far from the scattering region (or cluster) is a plane wave and therefore the same as the free particle state $\left|\psi_{k}\right\rangle$ in the absence of the potential, one can write the state $\left|\psi_{k}+\right\rangle$ applying time evolution operators to the free particle state $\left|\psi_{k}\right\rangle,\left|\psi_{k}+\right\rangle=\lim _{t \rightarrow-\infty} \hat{U}^{\dagger}(t) \hat{U}_{0}(t)\left|\psi_{k}\right\rangle$, where $\hat{U}_{0}(t)=e^{-i H_{0} t}$, and $\hat{U}(t)=e^{-i H t}$, where $\hat{H}=\hat{H}_{0}+\hat{V}_{L R}$, with $H_{0}$ as the tight-binding Hamiltonian of the model considered, whereas $V_{L R}$ describes the left and right lead connections to the Hamiltonian $\hat{H}_{0}$. Writing the previous result as the integral of its derivative, one has $\left|\psi_{k}+\right\rangle=\left[1+\left(\epsilon_{k}-H\right)^{-1} V_{L R}\right]\left|\psi_{k}\right\rangle$. In the case of the decoupled system of leads and ring, the state $\left|\psi_{k}\right\rangle$ for a particle in the left lead is a standing wave of the form $\sqrt{2 / \pi} \sin [k(j-1)]$, and therefore the amplitude of the state at site 0 is $\sqrt{2 / \pi} \sin k$ and the amplitude of the incident component of this standing wave is $1 / \sqrt{2 \pi}$. The $\left(\epsilon_{k}-H\right)^{-1} V_{L R}$ term acting on this state generates finite amplitude at site $N+1$ and this finite amplitude is constructed in the following way: (i) The amplitude at site 0 is $\sqrt{2 / \pi} \sin k$; (ii) the $V_{L R}$ term generates a hopping from the site 0 of the left lead to the site $L$ of the ring, and the respective hopping integral is $t_{L}$; and (iii) this finite amplitude propagates through both arms of the ring and reaches site $R$ of the ring, so that a finite amplitude is also present at site $R$. This process corresponds to the term $\left\langle R\left|\left[\epsilon_{k} \hat{I}_{s}-H_{s}+e^{i k}\left(t_{L}^{2}|L\rangle\left\langle L\left|+t_{R}^{2}\right| R\right\rangle\langle R|\right)\right]^{-1}\right| L\right\rangle$ in the previous equation which includes the effect of the coupling of the ring to the leads as modifications of the on-site energies of sites $L$ and $R$ ) the $V_{L R}$ term generates a hopping from the site $R$ of the ring to the site $N+1$ of the right lead, and the respective hopping integral is $t_{R}$. Since the transmission probability in the case of the open ring is given by the square of the absolute value of the ratio between the amplitude of the outgoing wave at site $N+1$ and the amplitude of the incident wave at site 0 , the transmission probability in Eq. (12) is obtained from the square of the absolute value of the product of these contributions.
The linear conductance is given by

$$
G(T)=\frac{e^{2}}{h} \int_{-B / 2}^{B / 2} d \epsilon\left(-\frac{d f}{d \epsilon}\left|t\left(\epsilon_{k}\right)\right|^{2}\right),
$$

where $f$ is the Fermi-Dirac probability function, given by $f(\epsilon)=\left[\exp \left(\frac{\epsilon-\mu}{k T}\right)+1\right]^{-1}$, where $\mu$ is the chemical potential, $k$ the Boltzmann constant, $T$ the temperature, and $B=1 / T$. For zero temperature one writes $G(0)=\frac{e^{2}}{\hbar}|t(\mu)|^{2}$.

In Fig. 6, the zero temperature conductance profiles for different choices of the central site orbital and of the lead contacts are shown in the case of six rings plus a central site, assuming hopping parameters $t_{1}=t=-0.17, t_{2}=-1, t_{R}=$ $t_{L}=0.1$, a varying chemical potential $\mu$ and three choices of angle for the contact sites 1 and 2. These plots indicate that a conductance experiment may serve as a probe of the existence of the central "edge" states. As is well known, transmittance peaks reflect a resonance effect when the energy of incoming particle is approximately equal to energy of one level of the cluster, but the width and height of the peak are also dependent on the probability density of the respective eigenstate at the contact sites. This becomes more evident assuming well-separated levels and considering the behavior of the transmittance very close to a level $\varepsilon_{\alpha}$,

$$
\begin{aligned}
T & =\left|t\left(\varepsilon_{k}\right)\right|^{2} \\
& \approx \frac{4 \tilde{t}_{L}^{2} \tilde{t}_{R}^{2} \sin ^{2}(k)}{\left[\varepsilon_{k}-\varepsilon_{\alpha}+\cos (k)\left(\tilde{t}_{L}^{2}+\tilde{t}_{R}^{2}\right)\right]^{2}+\sin ^{2}(k)\left(\tilde{t}_{L}^{2}+\tilde{t}_{R}^{2}\right)},
\end{aligned}
$$

for $\varepsilon_{k} \approx \varepsilon_{\alpha}$ with $\tilde{t}_{L}^{2}=t_{L}^{2}\left|\left\langle\varepsilon_{\alpha} \mid L\right\rangle\right|^{2}$ and $\tilde{t}_{R}^{2}=t_{L}^{2}\left|\left\langle\varepsilon_{\alpha} \mid R\right\rangle\right|^{2}$. This transmission peak has a Lorentzian-like curve, with a maximum at $4 \tilde{t}_{L}^{2} \tilde{t}_{R}^{2} /\left(\tilde{t}_{L}^{2}+\tilde{t}_{R}^{2}\right)$, indicating that there may be conductance peaks with $T<1$. The respective full width at half maximum (FWHM) is

$$
\mathrm{FHWM}=2 \sqrt{\tilde{t}_{L}^{2} \sin ^{2}(k)+\tilde{t}_{R}^{2} \sin ^{2}(k)} .
$$

In Fig. 6(b), one broad short peak is observed when the contacts are at the central state and a nearest-neighbor site. This peak occurs at the energy of the central "edge" state and the short height reflects the different probability density of the edge state at the contact sites. These short broad peaks can be seen also in the others plots of Fig. 6 reflecting in general a edge-state level or an impurity state (if the on-site energy of the central site pushes the edge state out from the respective band gap). Note that if both the effective hopping terms are small and different, then one observes a short narrow peak, so in order for a edge-state peak to be broad, at least one contact site must be at the central site.

In Figs. 6(b) and 6(c), two particular edge or impurity states are shown that generate two broad peaks. These states are edge states of seven-site and six-site $k_{\theta}=0$ chains respectively, since lowering the central site on-site energy (to $\varepsilon_{0}=$ 0 ) effectively decouples this site from the rest of the chain, and therefore the edge state has zero weight at the central site in Fig. 6(c). Similar behavior is observed in Figs. 6(d)-6(g).

In Fig. 7, similar conductance profiles are shown in the case of hopping parameters $t_{1}=t=-1, t_{2}=-0.17$, that is, the ratio between the $t_{1}$ and $t_{2}$ was reversed, which should bring the effective chains from the trivial to the nontrivial 


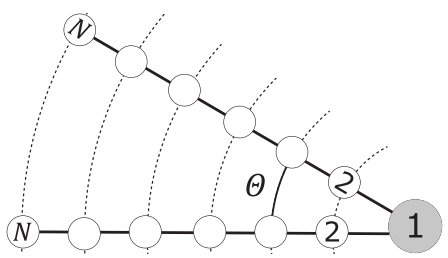

(a)



(b)



(d)

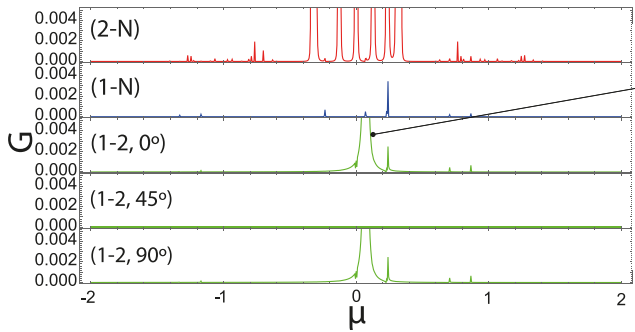

(f)


(c)


(e)


(g)

FIG. 6. Conductance profiles for our simplified radial model with six rings and a central site, assuming hopping parameters $t_{1}=t=-0.17$, $t_{2}=-1, t_{R}=t_{L}=0.1$, a varying chemical potential $\mu$ and temperature $T=0 \mathrm{~K}$. Different placement of the contacts is considered: For conductance profiles in red, contacts are placed in $2-N$ sites, in blue $1-N$ and in green $2-N$ [as shown in (a)], for this last case, angular dependence of the conductance is analyzed for angles $\theta=0^{\circ}, \theta=45^{\circ}$, and $\theta=90^{\circ}$ [as seen in (a)]. In the left-hand side, for (b), (d), and (f) the profiles are obtained assuming a central local potential $\varepsilon_{0} \neq 0$. (b) represents a $s$ orbital with $\varepsilon_{0}=2$, (d) a $p$ orbital with $\varepsilon_{0}=2$ and (f) $\varepsilon_{0}=-2$. In the right-hand side, for (c), (e), and (g) the profiles are obtained assuming a central potential $\varepsilon_{0}=0$. (c) corresponds to an $s$ orbital, (d) a $p$ orbital, and (f) a $d$ orbital. Schematic representation of states corresponding to different peaks in the profiles are shown to the right of each plot. Conductance profiles were obtained numerically with a resolution $\Delta \mu=0.005$.

topological phase and vice versa, leading to the disappearance or appearance of broad peaks.

The angular dependence of the conductance is also analyzed for contact placements 1 and 2 [see Fig. 6(a)]. As one can observe, for the conductance profiles with an $s$-orbital central site, no change of the conductance with the angle is observed. For the other cases, where a $p$ and $d$ orbital is considered, variations on the conductance peaks is observed. For the $p$-orbital central-site case, one observes that several peaks disappear for an angle of $90^{\circ}$, while for the $d$ orbital the same occurs, however, at a different angle, $45^{\circ}$. This is consistent with the different states shown next to the plots in Fig. 6. For example, let us consider the state, for a $d$-orbital central site, in Fig. 6(f). As one can see from the schematic representation of the state, for placements 1 and 2 , at the site corresponding to 2 and at an angle $\theta=45^{\circ}$, the probability density of the state at that site is zero, and therefore no conductance is expected.

\section{CORNER STATES}

In the previous sections, we have described a particular set of topologically protected states that appear at the center of a weblike cluster. These states reflect a mapping of the 


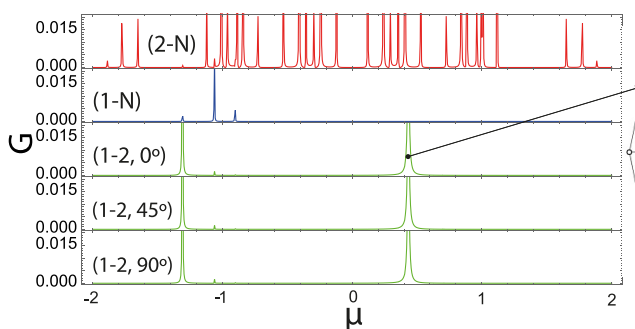

(a)



(c)

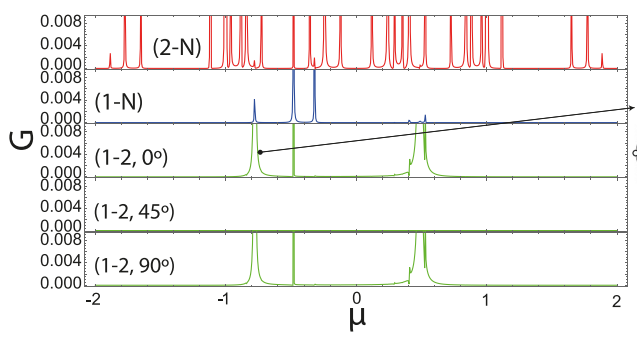

(e)
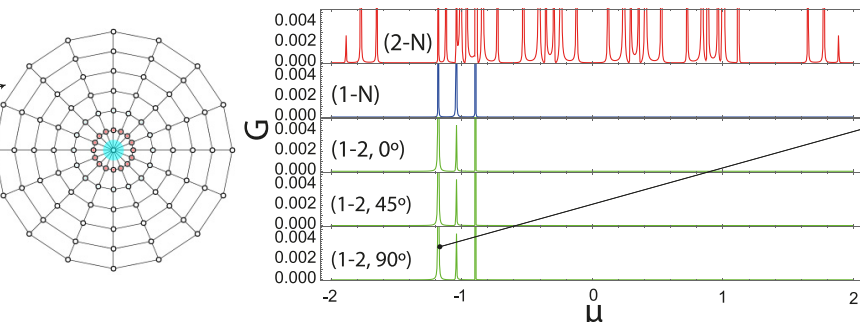

(b)


(d)
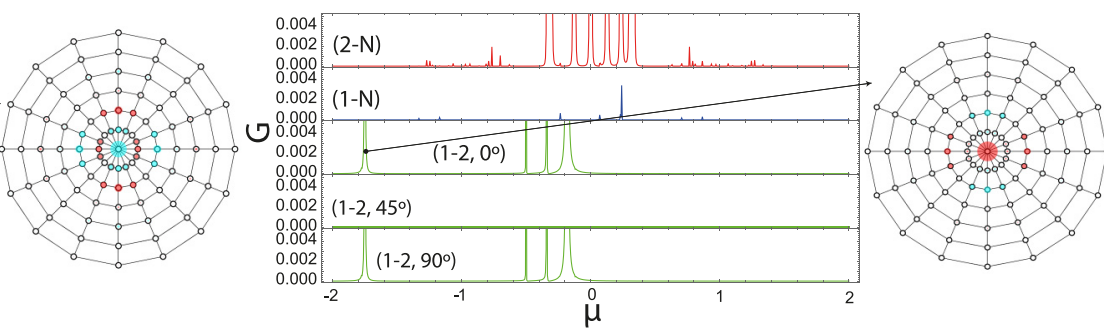

(f)

FIG. 7. Conductance profiles for our simplified radial model with six rings and a central site, assuming hopping parameters $t_{1}=t=-1$, $t_{2}=-0.17, t_{R}=t_{L}=0.1$, a varying chemical potential $\mu$ and temperature $T=0 \mathrm{~K}$. Different placement of the contacts is considered: For conductance profiles in red, contacts are placed in $2-N$ sites, in blue $1-N$ and in green $2-N$ [as shown in Fig. 6(a)], for this last case, angular dependence of the conductance is analyzed for angles $\theta=0^{\circ}, \theta=45^{\circ}$, and $\theta=90^{\circ}$ [as seen in Fig. 6(a)]. On the left-hand side, for (a), (c), and (e) the profiles are obtained assuming a central local potential $\varepsilon_{0} \neq 0$. Panel (a) represents an $s$ orbital with $\varepsilon_{0}=2$, (c) a $p$ orbital with $\varepsilon_{0}=2$, and (e) $\varepsilon_{0}=-2$. On the right-hand side, for (b), (d), and (f) the profiles are obtained assuming a central potential $\varepsilon_{0}=0$. Panel (b) corresponds to an $s$ orbital, (d) a $p$ orbital, and (f) a $d$ orbital. Schematic representation of states corresponding to different peaks in the profiles are shown to the right of each plot. Conductance profiles were obtained numerically with a resolution $\Delta \mu=0.005$.

tight-binding model of the cluster into decoupled SSH chains. We now consider staggered hopping terms not only in the radial direction but also in the azimuthal direction so that the weblike cluster can be interpreted as a 2D SSH lattice. It is well known that this 2D SSH lattice in the case of OBC in both directions displays zero energy corner states which are, however, buried within bulk bands. The zero energy gap can be opened by the introduction of a homogeneous magnetic flux in the lattice. For a $\pi$-flux per plaquette, the corner states were shown to be a consequence of a higher-order topological phase, whose quantized topological invariant is given by the quadrupole moment [31,32]. An alternative way of gapping the corner states, without requiring the inclusion of a magnetic flux, consists of assuming different ratios for the staggered hoppings along each direction [33].

In order to generate $\mathrm{OBC}$ in the azimuthal direction we consider a semicircular weblike cluster as shown in Fig. 8(a). The existence of the central site sets the difference between this cluster and the 2D SSH lattice. In the case of the 2D $\mathrm{SSH}$ lattice with an $s$ orbital at the central site, the corner states are almost degenerate (finite size effects lift slightly the degeneracy). In the case of the semicircular weblike cluster, only one of these corner states couples to the central site. The selection of this particular corner state is dictated by the distribution of the central site hopping terms that reflects the symmetry of the central site orbital. This is observed in Figs. 8(b), 8(d), and 8(f). Corner states that have different symmetry than that of the central site orbital do not combine with the central site orbital and their respective energy is not changed. As the central site energy is increased, the state that results from the linear combination of the central site orbital and the corner state with the same symmetry increases its energy and eventually leaves the band gap becoming an impurity state [see curves IV and V in Figs. 8(c), 8(e), and $8(\mathrm{~g})]$. Note that such transformation between an edge state and an impurity state was also observed in Ref. [26]. This impurity state can be interpreted as a linear combination of the central site orbital with a corner state but also with an edge state around the central site that has the same symmetry [see diagrams IV and V in Figs. 8(b), 8(d), and 8(f)]. 


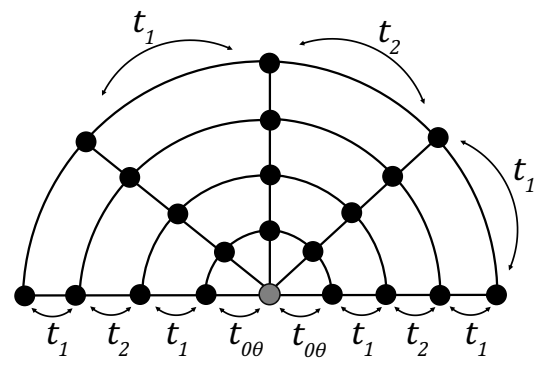

(a)

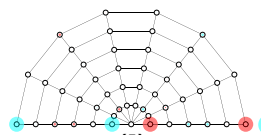

(I)

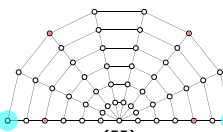

(II)

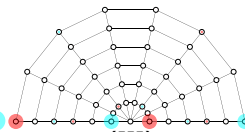

(III)



(IV)

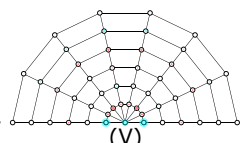

(v)

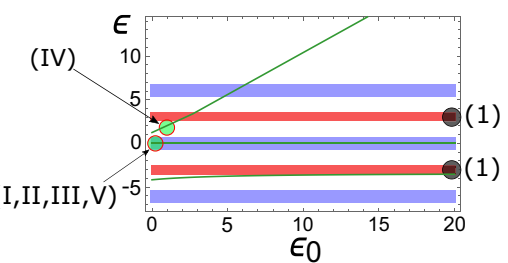

(c)

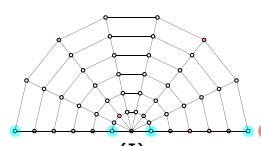

(I)

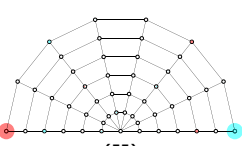

(II)

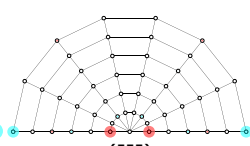

(III)

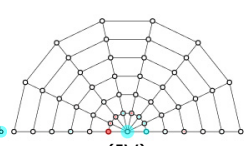

(IV)

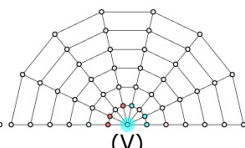

(V)

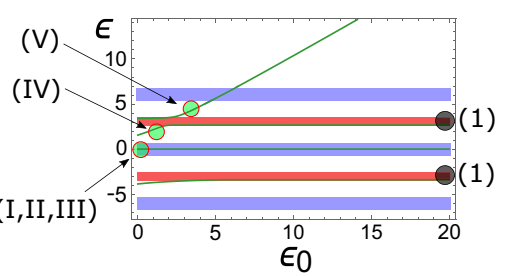

(e)

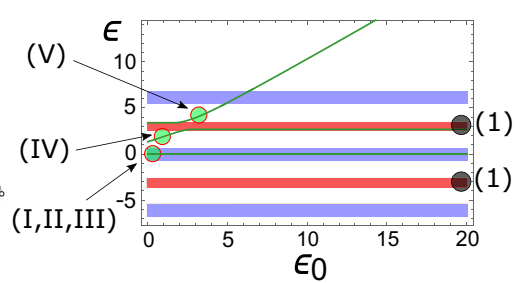

(g)

FIG. 8. (a) Schematic representation of the sliced structure. Staggered hopping parameters $t_{1}$ and $t_{2}$ are considered in both radial and azimuthal directions. Schematic representation of the observed corner states. For (b) the central site represents an $s$ orbital, (d) a $p$ orbital, and (f) a $d$ orbital. Staggered hopping parameters are assumed in both the radial and azimuthal directions, with $t_{1}=-0.5$ and $t_{2}=-3$, as seen in (a). It is assumed an on-site potential for the central site $\varepsilon_{0}=0$. In (c), (e), and (g) the respective spectra as a function of the on-site potential at the central site is shown. Topological edge-state energy bands are marked by black circles and labeled with (1).

\section{CONCLUSION}

In this paper, we studied a qualitative model of molecules with rotational symmetry with staggered hopping amplitudes in the radial direction and we have shown that the use of generalized coordinates allows a simple topological characterization of its behavior. In this qualitative model, the radial decay of the hopping parameters was neglected and the same number of sites was assumed in each concentric ring.

We have addressed in detail the dependence of the topological characterization of this weblike molecule on the central site local potential and orbital symmetry. Using the discrete rotational symmetry of the model and working with a basis of eigenstates of angular momentum, the model was mapped onto a set of effective SSH chains with an extra (central) site being respectively (a) an $s$ orbital, (b) a $p$ orbital, and (c) a $d$ orbital. The symmetry (we considered the cases of $s, p$, and $d$ orbitals) of the central site orbital determines to which effective chain the extra site is coupled.

One of our main conclusions is that single-particle excitations of this simplified model include central "edge" states besides the expected edge states at the boundary of the molecule. Internal edge states have also recently been found on fractal lattices [34-36]. These states are localized along the inner boundaries of the fractal holes and one can argue these are not truly in the bulk of the lattice like the central edge states described in this paper. More similar to our inner edge states are the internal edgelike states found in rotationally symmetric quasicrystals in the presence of a uniform magnetic field [37] which are localized in an internal effective edge of the quasicrystals. The reason why these states occur and their topological characterization is still an open problem [37]. It 
would be possible to follow in these systems an approach similar to that described in this paper if one chooses a circular gauge for the magnetic field (centered at the central site of the lattice) in order to preserve the rotational symmetry about this point. Curiously, in Ref. [37], it is stated that these internal edgelike states are robust against breaking the discrete rotational symmetry of the lattice.

Our weblike molecule falls into the weak topological insulators class but with contrasting features in what concerns the central "edge" states that imply that one cannot characterize its behavior using a single weak topological invariant as usually assumed [22]. The existence of these central "edge" states can be probed by conductance experiments since they lead to observable features in the conductance profiles for our simplified radial model. Introducing staggering of the hopping amplitudes also in the azimuthal direction and slicing the molecule, we have found that this sliced version of the model displays nonprotected zero-energy corner states buried within a bulk band. This is expected since this model can be interpreted as a $2 \mathrm{D} \mathrm{SSH}$ lattice and a magnetic flux is required in order to push these states into a band gap. However a particular corner state with a symmetry that mimics that of the central site orbital couples to the latter and it is driven into a band gap.

The topological features associated with the weblike geometry can be potentially probed in two experimental scenarios: (i) through the use of artificial lattices, as discussed in the Introduction, and (ii) in real molecules with some type of rotational symmetry. In this last scenario, while it is correct to say that one does not have control over the geometry or the tunneling parameters, one should recall that one of the most important properties of topological Hamiltonians is precisely the topological protection against perturbations that do not imply the closing of energy gaps. This implies that, for instance, our model will retain qualitatively its features even if the hopping integrals become smaller as one moves away from the center of the molecule. This is simple to understand when the ratio between $t_{1} / t_{2}$ is large since the central edge state will be strongly localized in this case and therefore determined by the hopping terms near the center. So, our model serves as a toy model to describe the qualitative behavior of molecules (in particular in what concerns the central "edge" states) that show approximately some discrete rotational symmetry and some staggering of the hopping integrals near the center of the molecule. Therefore, this discussion is relevant for instance to understand excited one-magnon states in very high-spin organic molecules with rotational symmetry.

\section{ACKNOWLEDGMENTS}

This work is funded by FEDER funds through the COMPETE 2020 Programme and National Funds throught FCTPortuguese Foundation for Science and Technology under the project PTDC/FIS-MAC/29291/2017, and was developed within the scope of the Portuguese Institute for Nanostructures, Nanomodelling, and Nanofabrication (i3N) Project No. UIDB/50025/2020 and UIDP/50025/2020. A.M.M. acknowledges financial support from the FCT through the work contract CDL-CTTRI-147-ARH/2018. R.G.D. appreciates the support by the Beijing CSRC. We thank S. T. Amin for helpful discussions.
[1] J. K. Asbóth, L. Oroszlány, and A. Pályi, A short course on topological insulators, in Lecture Notes in Physics (Springer, Berlin, 2016).

[2] A. M. Marques and R. G. Dias, Topological bound states in interacting $\mathrm{Su}-\mathrm{Schrieffer-Heeger} \mathrm{rings,} \mathrm{J.} \mathrm{Phys.:} \mathrm{Condens.}$ Matter 30, 305601 (2018).

[3] M. Leder, C. Grossert, L. Sitta et al., Real-space imaging of a topologically protected edge state with ultracold atoms in an amplitude-chirped optical lattice, Nat. Commun. 7, 13112 (2016).

[4] N. Parappurath, F. Alpeggiani, L. Kuipers, and E. Verhagen, Direct observation of topological edge states in silicon photonic crystals: Spin, dispersion, and chiral routing, Sci. Adv. 6, eaaw4137 (2020).

[5] S. Imhof et al., Topolectrical-circuit realization of topological corner modes, Nat. Phys. 14, 925 (2018).

[6] S. Liu, W. Gao et al., Topologically protected edge state in two-dimensional Su-Schrieffer-Heeger circuit, Research 2019, 8609875 (2019).

[7] N. A. Olekhno et al., Topological edge states of interacting photon pairs emulated in a topolectrical circuit, Nat. Commun. 11, 1436 (2020).
[8] C. Hua Lee et al., Topolectrical circuits, Commun. Phys. 1, 39 (2018).

[9] B. G.-g. Chen, N. Upadhyaya, and V. Vitelli, Nonlinear conduction via solitons in a topological mechanical insulator, Proc. Natl. Acad. Sci. USA 111, 13004 (2014).

[10] L.-Y. Zheng, V. Achilleos, O. Richoux, G. Theocharis, and V. Pagneux, Observation of Edge Waves in a Two-Dimensional Su-Schrieffer-Heeger Acoustic Network, Phys. Rev. Appl. 12 034014 (2019).

[11] R. Drost et al., Topological states in engineered atomic lattices, Nat. Phys. 13, 668 (2017).

[12] M. R. Slot et al., Experimental realization and characterization of an electronic Lieb lattice, Nat. Phys. 13, 672 (2017).

[13] C. Chin, R. Grimm, P. Julienne, and E. Tiesinga, Feshbach resonances in ultracold gases, Rev. Mod. Phys. 82, 1225 (2010).

[14] G. Pelegrí, A. M. Marques, R. G. Dias, A. J. Daley, V. Ahufinger, and J. Mompart, Topological edge states with ultracold atoms carrying orbital angular momentum in a diamond chain, Phys. Rev. A 99, 023612 (2019).

[15] L. Lin, Y. Ke, and C. Lee, Interaction-induced topological bound states and thouless pumping in a one-dimensional optical lattice, Phys. Rev. A 101, 023620 (2020). 
[16] Very high-spin organic molecules and polymers: Polyarylmethyl polyradicals, http://chemweb.unl.edu/rajca/highspin. html.

[17] J. Richard (ed.), Advances in Physical Organic Chemistry, 1st ed., Vol. 40 (Elsevier/Academic Press, Amsterdam, 2005), pp. $153-154$.

[18] A. Rajca, J. Wongsriratanakul, S. Rajca, and R. L. Cerny, Organic spin clusters: Annelated macrocyclic polyarylmethyl polyradicals and a polymer with very high spin $\mathrm{S}=6-18$, Chemistry 10, 3144 (2004).

[19] J. C. Slater and G. F. Koster, Simplified LCAO method for the periodic potential problem, Phys. Rev. 94, 1498 (1954).

[20] W. P. Su, J. R. Schrieffer, and A. J. Heeger, Solitons in Polyacetylene, Phys. Rev. Lett. 42, 1698 (1979).

[21] P. Delplace, D. Ullmo, and G. Montambaux, Zak phase and the existence of edge states in graphene, Phys. Rev. B 84, 195452 (2011).

[22] L. Fu, C. L. Kane, and E. J. Mele, Topological Insulators in Three Dimensions, Phys. Rev. Lett. 98, 106803 (2007).

[23] J. E. Moore and L. Balents, Topological invariants of timereversal-invariant band structures, Phys. Rev. B 75, 121306(R) (2007).

[24] R. Roy, Topological phases and the quantum spin Hall effect in three dimensions, Phys. Rev. B 79, 195322 (2009).

[25] A. M. Marques and R. G. Dias, Analytical solution of open crystalline linear 1d tight-binding models, J. Phys. A: Math. Theor. 53, 075303 (2020).

[26] C. W. Duncan, P. Öhberg, and M. Valiente, Exact edge, bulk, and bound states of finite topological systems, Phys. Rev. B 97, 195439 (2018).
[27] L. Banchi and R. Vaia, Spectral problem for quasi-uniform nearest-neighbor chains, J. Math. Phys. 54, 043501 (2013).

[28] D. Hügel and B. Paredes, Chiral ladders and the edges of quantum Hall insulators, Phys. Rev. A 89, 023619 (2014).

[29] A. M. Marques and R. G. Dias, One-dimensional topological insulators with noncentered inversion symmetry axis, Phys. Rev. B 100, 041104(R) (2019).

[30] T. Enss, V. Meden, S. Andergassen, X. Barnabé-Thériault, W. Metzner, and K. Schönhammer, Impurity and correlation effects on transport in one-dimensional quantum wires, Phys. Rev. B 71, 155401 (2005).

[31] W. A. Benalcazar, B. A. Bernevig, and T. L. Hughes, Electric multipole moments, topological multipole moment pumping, and chiral hinge states in crystalline insulators, Phys. Rev. B 96, 245115 (2017)

[32] F. Schindler, A. M. Cook, M. G. Vergniory, Z. Wang, S. S. P. Parkin, B. A. Bernevig, and T. Neupert, Higher-order topological insulators, Sci. Adv. 4, eaat0346 (2018).

[33] G. Pelegrí, A. M. Marques, V. Ahufinger, J. Mompart, and R. G. Dias, Second-order topological corner states with ultracold atoms carrying orbital angular momentum in optical lattices, Phys. Rev. B 100, 205109 (2019).

[34] M. Brzezińska, A. M. Cook, and T. Neupert, Topology in the Sierpiński-Hofstadter problem, Phys. Rev. B 98, 205116 (2018).

[35] S. Pai and A. Prem, Topological states on fractal lattices, Phys. Rev. B 100, 155135 (2019).

[36] A. A. Iliasov, M. I. Katsnelson, and S. Yuan, Hall conductivity of a Sierpiński carpet, Phys. Rev. B 101, 045413 (2020).

[37] C. W. Duncan, S. Manna, and A. E. B. Nielsen, Topological models in rotationally symmetric quasicrystals, Phys. Rev. B 101, 115413 (2020). 